\title{
Creators and Backers in Rewards-Based Crowdfunding: Will Incentive Misalignment Affect Kickstarter's Sustainability?
}

\author{
Michael Wessel \\ Copenhagen Business School \\ wessel@cbs.dk
}

\author{
Rob Gleasure \\ Copenhagen Business School \\ rg.digi@cbs.dk
}

\author{
Robert J. Kauffman \\ Copenhagen Business School \\ Singapore Management University \\ rk.digi@cbs.dk
}

\begin{abstract}
Incentive misalignment in rewards-based crowdfunding occurs because creators may benefit disproportionately from fundraising, while backers may benefit disproportionately from the quality of project deliverables. The resulting principal-agent relationship means backers rely on campaign information to identify signs of moral hazard, adverse selection, and risk attitude asymmetry. We analyze campaign information related to fundraising, and compare how different information affects eventual backer satisfaction, based on an extensive dataset from Kickstarter. The data analysis uses a multi-model comparison to reveal similarities and contrasts in the estimated drivers of dependent variables that capture different outcomes in Kickstarter's funding campaigns, using a linear probability model (LPM), which is a special case of the binary probability model. Our results reveal inconsistencies in funding information compared to backers' satisfaction, and a platform-wide trend of decreasing satisfaction. The findings broadly suggest fundraising is influenced by information disclosure and backer feedback, while eventual backer satisfaction is closely potentially caused by information about deferred compensation and long-term relationship-building.
\end{abstract}

\section{Introduction}

Crowdfunding platforms have become a popular means of launching new ventures, bringing together large crowds of potential investors (hereafter termed backers) around projects. The global crowdfunding market was valued at US\$13.9 bn in 2019 and is predicted to triple by 2026 [31]. Crowdfunding allows creators to access critical funding, while also helping them to find others with whom they share common interests [6]. Entrepreneurs (hereafter termed creators) are increasingly turning to crowdfunding, and not traditional financiers, because a well-executed crowdfunding campaign can help to create a strong community of backers for a new venture even before it is presented to the broader public [29]. So, entrepreneurs (hereafter termed creators), are turning to crowdfunding not traditional financiers, with a global crowdfunding 2025 market of US $\$ 28.8$ bn predicted.

The benefits of crowdfunding depend on projects receiving the resources they need. This explains why existing crowdfunding research has put emphasis on the fundraising process. Fundraising is only the first step. Once backers have committed the required funds, creators must deliver on the promises of the project. Yet rewards-based crowdfunding, where backers donate in return for future rewards, typically requires full downpayment by backers often months or even years in advance of the intended project outcomes. ${ }^{1}$

The arrangement between creators and backers is something mainstream management theory considers naïve. Crowdfunding platforms create natural conditions for goal, information, and risk asymmetries, though there is little formal governance to manage the resulting agency problem. Instead, backers must trust that creators have the skills and benevolence to act in their shared interests. Existing research has spent considerable effort exploring how creators build crowdfunding campaigns with characteristics to generate this trust [24]. However, that research has not addressed whether the trust generated by these characteristics is deserved. This is an oversight, due to an incentive misalignment that disproportionately rewards creators for attracting funds, even if they do not generate positive outcomes for backers.

Our study explores the problem of incentive misalignment in rewards-based crowdfunding. We performed a review of the rewards-based crowdfunding literature to identify campaign characteristics that predict fundraising success. We used a data-gathering process that yielded 377,778 fundraising campaigns from Kickstarter. They support comparisons of campaigns according to fundraising and eventual backer satisfaction. Our results show characteristics that predict fundraising, counterintuitively, do not predict backer satisfaction. More worryingly, several exhibit negative correlations, which require that we assess the robustness of the estimated models, and limitations in our empirical design.

\footnotetext{
${ }^{1}$ This is like a down-payment contract that assumes high trust [16].
} 
We also show trends toward increasingly effective fundraising, but decreasing backer satisfaction, and decreasing platform activity.

\section{Intellectual foundations for crowdfunding and incentive misalignment}

We next discuss several bodies of knowledge from Strategy, Economics, Management, Accounting and IS that offer intellectual foundations for this research.

\subsection{Rewards-based crowdfunding}

Crowdfunding platforms connect commercial, social, and artistic projects with potential backers, affording them an alternative to traditional sources of financing, such as banks or venture capitalists [27]. Different platforms facilitate different types of crowdfunding. Some focus on interest-based loans, and others on purchase of equity or donations [2]. Rewards-based crowdfunding has produced disruptive outcomes, from cultural projects to new technologies though.

Rewards-based crowdfunding appeals to creators and backers for a variety of reasons. It allows mutuallybeneficial short-term financial benefits - creators get revenue at a time of need, and backers get valuable discounts or assets. Crowdfunding also can contribute to viral marketing and generate interest among potential consumers and user groups. And some backers and creators believe the grass-roots approach is more likely to create shared value with wide-ranging social benefits [18]. Policy-makers encourage the growth of these platforms, building on evidence that crowdfunding campaigns boost innovation [32], stimulate local economies, and create opportunities for entrepreneurs [20].

These benefits assume crowdfunded projects can deliver their promised rewards. This has been controversial though. Many commentators have demonstrated why crowdfunding projects disappoint backers, with the risk of fraud [10], inexperience or poor planning creating delays or unforeseen problems [30], and systemic coordination and communication challenges as projects grow in size [22]. All of these assume creators are at fault. However, it is also possible backers are disappointed because of unrealistic expectations, or because they misunderstood the intentions of the project. ${ }^{2}$

Crowdfunding differs from other forms of crowdsourcing in two notable ways. First, the crowd in crowdfunding platforms do not usually participate directly in the co-creation process. Instead, they entrust

\footnotetext{
${ }^{2}$ The threat of backers becoming dissatisfied is not only a problem for projects. It may harm crowdfunding platforms as a whole, if backers become disillusioned over time. For instance, platform-wide funding activity may decrease when individual campaigns are suspended [10].

${ }^{3}$ An agent takes actions and make decisions to reflect the interest of an invested principal in the presence of some information asymmetry.
}

design and development to the creator, limiting the role of backers to onlookers who only make suggestions [15]. Second, the crowd must commit their support to a project at the outset, not when they are satisfied with the project's outcome. So, they are committing to risky outcomes subject to change.

\subsection{The incentive-misalignment problem}

This type of arrangement is a principal-agent relationship. ${ }^{3}$ It creates conditions wherein the goals of the principal and agent diverge and the principal has limited means to verify the agent is acting appropriately. Eisenhardt [11] notes three related issues: (1) moral hazard, where the agent can pursue opportunistic and selfish behaviors invisible or poorly understood by the principal (2) adverse selection, where the agent presents to the principal as being more capable than they actually are; and (3) risk attitude asymmetry, where the principal and the agent may prefer contrasting actions because they are impacted differently by risk.

Rewards-based crowdfunding is susceptible to incentive misalignment. A fundraising campaign is a contract of sorts, but backers cannot negotiate specific commitments with creators. They provide funding at the project start and have little legal ability to enforce penalties or rewards once the campaign is underway. ${ }^{4} \mathrm{~A}$ counterargument suggests this structure is not problematic though. Backers and creators are invested in the desirability of the project. However, previous management research suggests that when principals lack the ability to monitor agents' activities, the sharing of short-term benefits may have an adverse effect [26]. It may encourage agents to use the principals' resources to maximize short-term earnings. ${ }^{5}$

A benefit for creators is the ability to gauge demand for subsequent products and services, rather than make premature long-term commitments [2]. Backers lack this ability, as they have already committed their resources. This leaves them at the mercy of creators, should the latter choose to leverage the backers' funds for maximum short-term gain.

Crowdfunding makes creators focus disproportionately on fundraising (which may not impact backers, if a project has met its targets) and backers to focus on outcome quality (which may not benefit creators). So how, then, can principal-agent relationships deal with incentive misalignment? We consider four strategies.

It allows the agent to hide certain actions and information.

${ }^{4}$ This is true even if creators have delivered rewards, and backers are upset with the quality, timeliness, customer support, and discounts relative to subsequent consumer releases [4].

${ }^{5}$ Agents can walk away from a long-term relationship, so they weigh short-term benefits and long-term costs / risks, which is not abstract. 


\subsection{Contracts, disclosures, and compensation}

A basic means to address these issues is to align the incentives between principals and agents, so each works toward the same outcomes [26]. Principals, first, do this with a contracting strategy. They allocate some or all of the reward to an agent, based on adherence to some behaviors and outcomes.

This is complicated though: the goals specified for complex endeavors are often vague, evolving, and even paradoxical [5]. Crowdfunding campaigns are especially vulnerable to these challenges. The projects are often early-stage and backers are less knowledgeable and experienced than traditional investors are. This makes it challenging to build a shared understanding for specifying the goals - let alone balancing an appropriate portfolio of incentives.

Information asymmetry is at the heart of incentive misalignment, and information disclosure, thus, is valuable: detailed communication will make it difficult for a principal to mislead an agent. The second, a voluntary disclosure strategy, is especially powerful: managers and insiders cannot hide information to obtain more trust from financiers [17].

The manner in which creators make voluntary disclosures can vary though. For example, creators can communicate via offline relationships or social media [33]. Yet most crowdfunding campaigns take place on platforms that act as shared spaces for different groups of backers. Collectively, they rely heavily on information, and creators choose to provide it within the platform to evaluate projects [34]. Thus, it is unsurprising that voluntary disclosure on crowdfunding platforms may influence fundraising success (though excessive disclosure may become problematic) [25].

The third is a feedback strategy for managing incentive misalignment. Feedback is a key component of monitoring activities in agency relationships. It allows the principal to express encouragement or dissatisfaction to the agent. This helps an agent gauge the intent and priorities of the principal. And, it allows the agent to judge the effectiveness of their communication and respond to the principal in iterative negotiation [15].

Feedback appears especially important in crowdfunding environments for four reasons. First, the limited monitoring activities available to backers mean they must continuously request and validate information being exchanged [14]. Second, the community of backers is likely to have a more diverse and unpredictable set of interests than traditional investors [27], creating uncer-

\footnotetext{
${ }^{6}$ For knowledge-intensive agents, such opportunities can create a link between the tasks an agent is performing and their social identity. This deepens the agency relationship, assuming the agent will continue to perform the role in the future. Indeed, the goal of retaining an agent
}

tainty about those backers' expectations. Third, individual crowd members often lack the knowledge of experts [21]. So, they rely on collective judgments to spot problems. This informs their judgments of legitimacy with observed feedback from other backers [12]. Fourth, many backers attach themselves to projects because of group identity, and this feedback enables shared meaning to be created [1].

A fourth means, the deferred compensation strategy, incentivizes the agent to expend effort and not act opportunistically or selfishly. This is done by sharing the costs of missing the principal's goals (e.g., with delays) [23], or sharing the benefits of meeting its goals (e.g., via firm or product performance) [34]. Signaling intent is an important part of trust building in agency relationships, particularly in the early stage [8]. In addition to signaling competency and honesty, new ventures often wish to communicate strategic attitudes such as autonomy, competitive aggressiveness, and a willingness to take risks [28].

Deferred compensation, thus, is a way for the principal to manage risk; and it is a way for the agent to demonstrate confidence and commitment to long-term outcomes. Deferred compensation can take many forms but perhaps the most common includes developing additional long-term opportunities, such as career development [13]. ${ }^{6}$ This deferred compensation is also evident in crowdfunding projects, whereby fundraising may be affected by the perceived social identity of the creator and continued engagement on the platform [36].

\section{Context and methods}

\subsection{Research context: Kickstarter}

We collected and analyzed data from the rewardsbased crowdfunding platform, Kickstarter. As of mid2020 , US $\$ 5$ bn was raised to fund more than 182,000 projects (www.kickstarter.com). When creators set up their campaigns, they decide in which of Kickstarter's 15 categories (Art, Fashion, Games, and Technology) to launch them and set their funding goals. The latter indicates how much funding they need to move forward with their project. Kickstarter follows an all-or-nothing funding model, so only campaigns that reach the predefined funding threshold are considered to be successful and receive the collected funds. If the threshold is not reached, all backers will be reimbursed. Only about onethird of all its projects succeed.

Interactions among project creators and backers are publicly visible. ${ }^{7}$ We were able to trace them from a

can motivate offering deferred compensation.

${ }^{7}$ For example, if a creator succeeds in collecting sufficient funding, they must deliver on promises given to backers. The funding deadline initiates a work-intensive time for creators when they stay in touch 
campaign's launch until after rewards were to be delivered to backers. This high transparency makes Kickstarter the ideal context for studying the effects of incentive misalignment in reward-based crowdfunding. We could observe funding success - the outcome that benefits creators most - as well as whether backers were satisfied with the rewards delivered to them.

\subsection{Dataset}

We gathered a time-series dataset with a self-developed web crawler that covers the period from the launch of Kickstarter in April 2009 until April 2020. This initial dataset includes all 470,000 projects that ran on Kickstarter during that period. We limited our dataset in several ways:

(1) We excluded campaigns that did not provide an estimated delivery date for any offered rewards. This defines the start date for the period in which backers expressed their satisfaction or dissatisfaction with the rewards they were to receive. ${ }^{8}$

(2) We eliminated campaigns canceled by the creator or suspended for any reason by the platform.

(3) Nor did we include those with unavailable essential information (e.g., project descriptions).

Our final dataset has 377,778 campaigns, out of which 158,865 were successful. It contains basic information on all campaigns such as the funding goal, but also the text of more than $20 \mathrm{~mm}$ comments written by backers about these projects. (See Table 1. Descriptive statistics are in Appendix Table A.)

\subsection{Measures}

Dependent variables. There are two. We use a dichotomous variable for funding success, indicating whether a campaign has reached its funding goal. FundSucc is 1 if the project succeeded in reaching its funding goal and 0 otherwise.

We measure BackerSatisf by evaluating the valence of comments by backers indicating their satisfaction after the designated reward delivery date and for period of up to 6 months after the final reward was supposed to be delivered - the reward delivery period. A total of 5.6 $\mathrm{mm}$ comments were written by backers, an average of 15 comments per campaign (s.d. = 259). We examine their sentiment of these comments using the Valence Aware Dictionary and Sentiment Reasoner (VADER), a sentiment analysis tool to analyze social media text. ${ }^{9}$ To obtain our measure, BackerSatisf, from VADER, we

with backers through Kickstarter and update them on progress toward the delivery of rewards. In turn, backers can write to inquire about project status, or deviations in the time of reward delivery.

${ }^{8}$ Providing this information on Kickstarter was possible for creators from August 1, 2011 onwards. We only included campaigns for which we could observe a period of six months after the last rewards were supposed to be delivered. first derived a normalized weighted composite sentiment score for each of the 5.6 mm relevant comments. ${ }^{10}$ We then took the average of these scores per campaign.

Table 1. Kickstarter's campaign characteristics

\begin{tabular}{l|l|l}
\hline Strategy & Variables & Description \\
\hline \multirow{4}{*}{$\begin{array}{l}\text { Contract } \\
\text { design }\end{array}$} & FundGoal & All-or-nothing project target (\$) \\
& $\begin{array}{l}\text { TimeDeliv } \\
\text { \#Rewards }\end{array}$ & \# days for fundraising \\
\hline Feedback & \#acker comm & \# different reward levels offered \\
& Backer senti & \# comments made by backers \\
& \#ggregate score for sentiments \\
\hline \multirow{5}{*}{$\begin{array}{l}\text { \#eferred } \\
\text { compen- }\end{array}$} & BackedOthers & \# updates made by creator \\
sation & \# other projects creator backed \\
& PastProj & \# comments by creator \\
& Authenticity & \# prior projects by creator on platform \\
& FBProfile & Words to convey authenticity in descript. \\
& Cideator linked to Facebook profile (0/1) \\
\hline \multirow{5}{*}{$\begin{array}{l}\text { Info } \\
\text { disclosure }\end{array}$} & \#Images & Campaign had a video (0/1) \\
& Readability & \# images/videos in descript \\
& SpellingErrors & Text readability in descript \\
& \#Words & Description contains spelling errors (0/1) \\
& \#umTerms & Proportion numeric terms in descript \\
\hline \multirow{5}{*}{ Controls } & Category & Project Kickstarter category \\
& Year & Year launched \\
& StaffPick & Creator is located in the U.S. (0/1) \\
& Platform has project on homepage (0/1) \\
\hline
\end{tabular}

Independent variables. Past research has examined how different campaign characteristics predict backer commitment during the fundraising process. Yet, despite the likelihood of incentive misalignment, the extent to which the same campaign characteristics predict longer-term project outcomes remains unclear. We, thus, performed a systematic literature review to ensure our characteristics comparisons make sense.

We define the review scope as identifying and synthesizing the campaign characteristics connected with fundraising in rewards-based crowdfunding. We further conceptualize the topic as those campaign characteristics that are partially or totally visible to backers during the fundraising process. Rather than exploring and interpreting the different qualities that vary according to project context, we also limit the focus to empirical studies that perform quantitative analyses of multiple projects. For our literature search process, we wanted to include studies from a range of disciplines that had achieved a high level of methodological scrutiny. We searched the fifty journals included in the list provided by the Financial Times and used to rank international business schools (the “FT50”). We limited our search for January

\footnotetext{
9 VADER matches text against 7,500+ words, acronyms and initialisms as well as emojis - with sentiment polarity and intensity scores that have been determined with the help of human raters. This lexicon-based approached outperforms machine learning, especially for shorter texts that can be found on social media.

${ }^{10}$ This uni-dimensional measure of sentiment ranges between -1 (most negative) and +1 (most positive).
} 
2010 to January 2020. ${ }^{11}$ Search terms included keywords that could appear anywhere in an article ("crowdfunding" or "crowd funding") and popular platform names ("kickstarter" or "indiegogo"), in case empirical studies of such platforms were performed in other research streams and the authors chose not to refer to the platform type. This initially ,produced 132 articles from 22 of the 50 journals. ${ }^{12}$ We further harvested 63 articles on rewards-based crowdfunding, but only 40 quantitative empirical studies of rewards-based crowdfunding remained after more legitimacy checks. ${ }^{13}$

The literature review and analysis used a conceptcentric matrix to identify and synthesize common campaign characteristics linked to fundraising. This resulted in an initial set of 53 campaign characteristics with demonstrated correlations with fundraising. In the interests of parsimony, these were refined to remove characteristics that met any of the following exclusion criteria (1) they described qualities of the creator, often studied to observe bias and prejudice (e.g. age, ethnicity) (2) they were theoretical outliers that had not received widespread attention (e.g. rhetorical signals, anonymity) (3) they described the interaction between the campaign and other social contexts. Each would have to be analyzed separately (e.g., by location, early contributions from offline networks, social media interactions). This resulted in 20 characteristics grouped by the four strategies identified for managing incentive alignment (contract design, information disclosure, feedback, and deferred compensation).

Campaigns capture contract design by specifying the financial target as the collective investment required over some specific period. This is important for contracts: connecting investment directly to outcomes is important to persuade investors to prioritize particular projects. Hence, contracts also include a number of rewards which specify the different deliverables that individual backers can expect. While the details of different rewards vary, the number of rewards can be used to indicate the breadth of commitments made to backers. The timeline for the return of investment is also important in contract design [3]. Campaigns may plan to deliver different rewards by different dates, and the amount of time until their delivery is useful to assess project duration.

The manner in which campaigns capture feedback is perhaps the straightforward to measure. First, campaigns can capture feedback via the number of com-

\footnotetext{
${ }^{11}$ This avoided studies when crowdfunding was still emerging.

${ }^{12} 50$ articles removed were not focused on crowdfunding, another 10 focused on equity crowdfunding, 8 on P2P lending, and 1 on charity.

${ }^{13} 11$ were non-empirical, 10 were on macro-impacts of crowdfunding platforms, and another 2 were qualitative. All were screened out.

${ }^{14}$ Due to space limitations, we suppressed citations on some variables and measures well-known in crowdfunding research. The amount of
}

ments made during fundraising, which gives an indication of the amount of feedback and the willingness of backers to provide it. Second, they capture its significance via the extent of positive or negative sentiments. for encouragement or reproach [9].

Campaigns provide information disclosures and capture deferred compensation in multiple ways. ${ }^{14}$ First, the creator's participation in other projects shows longterm commitment to a community. This is indicated via the number of previous projects they have created and backed, or the number of comments they have left. This is further indicated by the creator's willingness to embed their personal identity in the project, by linking to a Facebook account [18] or using authentic language [36]. The characteristics increase perceptions the creator will have reputational benefits or costs from the project long after the campaign has finished.

Control variables. We incorporated project-level variables in our model to account for alternative explanations. First, dummy variables represent in which of the 15 main categories on Kickstarter creators launched their campaigns. Second, our dataset spans 2011 to 2019, so we included year dummies for when a campaign was launched to control for unobservable timevarying effects of changing platform dynamics. Third, we coded for campaign location in the U.S. (US). Finally, when we estimated BackerSatisfaction, we also included FundingPercentage, because raising more funds than the target requires producing more rewards, which explains why creators struggle with this process.

\subsection{Estimation by linear probability methods}

As our dependent variables are binary indicators, we estimate linear probability models (LPMs), a special case of the binary probability model. Though logit (logistic) and probit are standard for the analysis of binary dependent variables, LPMs have distinct advantages in our setting. Coefficients derived from logit and probit regression cannot easily be compared between models that differ in their dependent and independent variables. They also have unobserved heterogeneity. We are interested in the signs and significance levels and comparing them for the dependent variables, and LPMs are appropriate for interpreting such probability change results. Thus, a coefficient can be interpreted as the change in the probability that the dependent variable is equal to 1 , all else constant. We use heteroscedasticity-robust

information is indicated by the word count in a campaign description, while its depth is proxied by the proportion of numerical terms used. The number of updates provides ongoing streams of campaign information. And the ease that such information is understood is due to project description readability (Flesch-Kincaid) and the presence of spelling errors. Visual information, finally, is captured by the presence of a video and number of images, which offer content cues. 
standard errors to avoid predicting out-of-range probability, such as $p>1$ or $p<0$.

We first estimated the LPM specification using FundSucc (Model 1), and repeated it for the alternative dependent variable by replacing dependent variable with BackerSatisfact (Model 2). Besides the dependent variable, the models only differed with regard to the control variable for funding percentage, \%Fund, that we only included when the dependent variable was BackerSatisf. We checked the variance inflation factors (VIF) for potential multicollinearity. All VIF values for our independent variables matched recommended threshold values and mean VIF score requirements.

\section{Main Results}

The number of campaigns per month varied on Kickstarter between 2009 and 2019. (See Fig. 1) The spike in 2014 is attributable to a policy change that removed the mandatory screening by Kickstarter before a campaign's launch. Since 2015, the number of campaigns per month has been declining and is now on a similar level as it was in 2011.

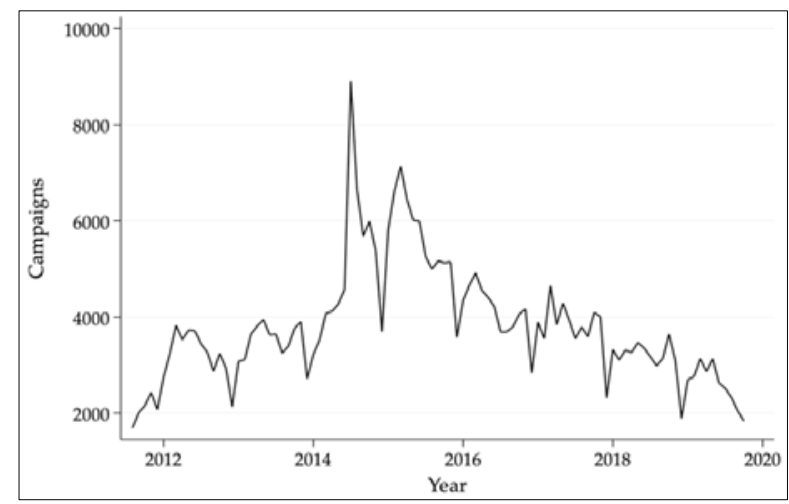

Fig 1. Number of campaigns, 2011-2019

FundSucc and BackerSatisf fell until 2014, when Kickstarter removed the mandatory campaign screening policy. After this, FundSucc grew steadily from $\sim 30 \%$ to $\sim 60 \%$ in 2019, while BackerSatis continued to decline, leading to a widening gap between both measures. (See Fig. 2.) The incentive misalignment may be the cause of these longer-term problems for Kickstarter, a relationship that deserves further investigation.

We assessed significance of differences for coefficients of the main models with this general statistic [7]:

$$
z=\left(\beta_{\mathrm{M} 1}-\beta_{\mathrm{M} 2}\right) / \sqrt{S E_{\beta_{\mathrm{M} 1}}^{2}+S E_{\beta_{\mathrm{M} 2}}^{2}}
$$

\footnotetext{
${ }^{15}$ Since we do not use the robust SEs for estimating the changes in the dependent variables, but only their differences (and page space is
}

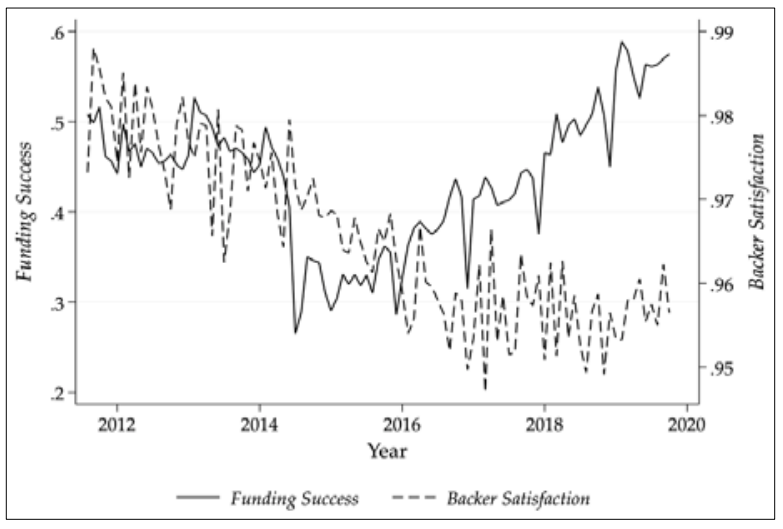

Fig 2. FundSucc and BackerSatisf, 2011-2019

The LPM results are shown in Table 2.

Table 2. LPM results for Models 1 and 2 (M1, M2)

\begin{tabular}{|c|c|c|c|}
\hline VARIABLE & $\begin{array}{c}\text { FundingSucc } \\
\text { (M1) }\end{array}$ & $\begin{array}{c}\text { BackerSatisf } \\
\text { (M2) }\end{array}$ & DIFF (z) \\
\hline \multicolumn{4}{|c|}{ Contract design } \\
\hline FundGoal & $-0.073^{* * *}$ & $0.003^{* * *}$ & $-123.58^{* * *}$ \\
\hline Duration & $-0.002^{* * *}$ & -0.000 & $-33.94^{* * *}$ \\
\hline TimeDeliv & $-0.000^{* * *}$ & $-0.000^{* * *}$ & $10.202^{* * *}$ \\
\hline \#Rewards & $0.034^{* * *}$ & $-0.012^{* * *}$ & $29.383^{* * *}$ \\
\hline \multicolumn{4}{|c|}{ Information disclosure } \\
\hline$\overline{\text { Video }}$ & $0.036^{* * *}$ & $0.003^{*}$ & $17.613^{* * *}$ \\
\hline \#Images & $-0.010^{* * *}$ & $-0.003^{* * *}$ & $-6.885^{* * *}$ \\
\hline Readability & $-0.001^{* * *}$ & -0.000 & $-18.788^{* * *}$ \\
\hline SpellErrors & $-0.037^{* * *}$ & $-0.005^{* *}$ & $-12.325^{* * *}$ \\
\hline \#Words & $0.008^{* * *}$ & $0.005^{* * *}$ & $3.486^{* * *}$ \\
\hline NumTerms & $0.001^{* * *}$ & $0.001^{* *}$ & $2.019^{* * *}$ \\
\hline \multicolumn{4}{|l|}{ Feedback } \\
\hline BackerComm & $0.012^{* * *}$ & $0.002^{* *}$ & $11.277^{* * *}$ \\
\hline BackerSentim & $0.082^{* * *}$ & $0.006^{* * *}$ & $27.334^{* * *}$ \\
\hline \multicolumn{4}{|c|}{ Deferred compensation } \\
\hline \#Updates & $0.207^{* * *}$ & $0.003^{* * *}$ & $207.352^{* * *}$ \\
\hline BackedOthers & $0.065^{* * *}$ & $0.008^{* * *}$ & $31.373^{* * *}$ \\
\hline CreatorComm & $0.226^{* * *}$ & $-0.028^{* * *}$ & $96.293^{* * *}$ \\
\hline PastProj & $0.006^{* * *}$ & $-0.003^{* *}$ & $4.713^{* * *}$ \\
\hline Authenticity & $-0.001^{* * *}$ & $0.000^{* * *}$ & $-17.085^{* * *}$ \\
\hline FBProfile & $-0.017^{* * *}$ & $0.004^{* * *}$ & $-14.813^{* * *}$ \\
\hline \multicolumn{4}{|c|}{ Control variables } \\
\hline Category & Incl. & Incl. & - \\
\hline Year & Incl. & Incl. & - \\
\hline US $(0 / 1)$ & $0.004^{* *}$ & 0.001 & $2.971^{* * *}$ \\
\hline StaffPick & $0.099^{* * *}$ & 0.001 & $42.435^{* * *}$ \\
\hline$\%$ Fund & & $0.007^{* * *}$ & - \\
\hline Constant & $0.765^{* * *}$ & $0.947^{* * *}$ & - \\
\hline$\overline{R^{2}}$ & 0.56 & 0.02 & - \\
\hline Obs. & 377,778 & 158,865 & - \\
\hline
\end{tabular}

Notes. ${ }^{* * *} p<0.01 ;{ }^{* *} p<0.05 ;{ }^{*} p<0.1$; robust SEs suppressed due to space limitations.

Since FundSucc and BackerSatisf were scaled equally, unstandardized $\beta$ coefficients are compared. The SE of the difference is the square root of the sum of the two squared SEs. ${ }^{15}$

limited), we chose to not present the SEs in Table 2, and we only discuss the $\beta$ estimates and their significance levels in this section. 
Regarding the contract design, the first inherent misalignment between the goals of creators and backers is evident from the opposing coefficients for funding goal. Logically, the estimate of higher funding goals suggests a lower probability of getting funded $\left(\beta_{\text {FundGoal_M1 }}=\right.$ 0.073, $p<0.001$ ), but also a higher probability for backer satisfaction $\left(\beta_{\text {FundGoal_M2 }}=0.003, p<0.001\right)$. Opposing coefficients can also be observed for the number of rewards offered. A higher number of rewards suggests a higher probability of funding ( $\beta_{\# \text { Rewards_M1 }}=$ $0.034)$, but also with a lower probability for backer satisfaction $\left(\beta_{\# \text { Rewards_M2 }}=-0.012, p<0.001\right)$.

For information disclosure, all coefficients align in direction across the two models, indicating that these signals do indeed help backers to discern the true quality of the respective project. For instance, a campaign that includes a video is more likely to get funded ( $\beta_{\text {Video_M1 }}=$ $0.036, p<0.001$ ) and also more likely to satisfy backers $\left(\beta_{\text {Video_M2 }}=0.003, p<0.05\right)$. Similarly, a longer project description has the same positive effect for funding and backer satisfaction, suggesting more detailed descriptions reduce the creator-backer information asymmetry.

Backer comments (BackComm) during the funding period also potentially cause both a higher probability to get funded (M1) and deliver more BackerSatisf (M2).

Table 3. Summary of misalignment between FundSucc and BackerSatisf

\begin{tabular}{l|c|c}
\hline VARIABLE & Funding Succ & Backer Satisf \\
\hline Contract design & - & + \\
\hline FundGoal & - & 0 \\
Duration & + & - \\
\#Rewards & \multicolumn{1}{|}{} \\
\hline Information disclosure & 0 \\
\hline Readability & - & - \\
\hline Deferred compensation & - \\
CreatorComm & + & + \\
PastProj & + & + \\
Authenticity & - & - \\
FBProfile & - &
\end{tabular}

Finally, for deferred compensation, we again observed some opposing coefficients. For instance, if creators engage in a discussion with backers during the funding period (by writing and responding to comments of backers, this was observed to promote a higher probability of getting funded ( $\beta_{\text {BackerComm_M1 }}=0.226, p<$ 0.001 ), but a lower probability for backer satisfaction $\left(\beta_{\text {BackerComm_M2 }}=-0.028, p<0.001\right)$. Similarly, experienced creators who launched at least one project on Kickstarter before were more likely to receive sufficient funding, but less likely to satisfy their backers. Interestingly though, we observed the opposite effect if creators

\footnotetext{
${ }^{16}$ We found that, based on research methods from Marketing (alternative market share estimation approaches) and Healthcare (patient healthcare expenditure driven by insurance status), typically limited dependent variables are preferred, but in Sociology (parental income
}

disclosed their Facebook profiles, which reduced their probability to get funded ( $\beta_{\text {PastProj_M1 }}=-0.017, p<$ $0.001)$, but increased the probability to eventually satisfy backers $\left(\beta_{\text {PastProjM2 }}=0.004, p<0.001\right)$.

\section{Robustness Assessment}

To check for the robustness of our results, we conducted further analyses. Though LPMs offer advantages in our setting, especially cross-model coefficient comparisons and a binary variable (with a binomial datagenerating distribution), our main results may be biased because the predicted values for the dependent variable are not arrived at in the same manner as those for limited dependent variable models. ${ }^{16}$ Thus, we conducted additional robustness testing to see what issues arose, and may affect the truth behind our estimates.

For our first check, we ran our analyses for FundSucc and BackerSatisf again, using a logit model (which yielded pseudo- $R^{2}$ s of 0.56 and 0.06 for M1 and M2). ${ }^{17}$ The results are consistent with those of the LPM in terms of the direction and significance of coefficients with one exception: the positive coefficient for PastProj became negative in one model. Second, bias may have arisen in our analysis because BackerSatisf was estimated on a partial dataset (i.e., all campaigns that were successful and expected to deliver rewards to backers).

To reduce the overlap between the datasets for our two models, we extracted two random samples of 100,000 campaigns and re-estimated the models. The estimated coefficients from this robustness check aligned with those of our main model for sign directions, magnitudes, and significance levels with two exceptions. The effect for BackerSenti and PastProj lost significance in M1. Second, given the changing dynamics on Kickstarter after the prescreening of campaigns was discontinued in June 2014, we reran our models based on a dataset with only campaigns launched after the policy change, so 110,323 campaigns were dropped. The coefficients again were consistent with our main model, with the exception of TimeDeliv, which became insignificant in M1 also. We conjecture that reframing this research in a new quasi-experimental design that emphasizes causal inference may lead to resolution of the conflicts and possible biases we observe.

\section{Discussion}

This study explored incentive misalignment in rewards-based crowdfunding. Using a large sample of projects from Kickstarter, we compared whether the

effects on high school graduation) and Criminology (delinquent peers' effects on young people's incarceration), LPMs with binary dependent variables are common and encouraged.

${ }^{17}$ We discuss some possible reasons for M2's weaker fit at the end. 
types of information involved with fundraising are also linked to eventual backer satisfaction. Our results identified inconsistencies in these relationships and suggested this may be due to deteriorating platform participation - an unexpected observation. The findings thus make several contributions to crowdfunding research.

First, many previous studies have explored the role of campaign information in attracting funding. Crowdfunding exposes backers to disappointment though, due to weak approximation of the legal contract from campaign information. To our knowledge, this is the first study to connect the two literature streams at the campaign information level. This is a step toward explaining agency relationships in rewards-based crowdfunding.

Second, we identified types of information that appear consistent and inconsistent. Some types of campaign information have a positive relationship with both fundraising and eventual backer satisfaction. These types are thought of as being connected with information disclosure and feedback. Other types have significant relationships with fundraising but not eventual backer satisfaction, especially readability, funding duration, and staff picks. These relationships are difficult to interpret, as the lack of significance may simply be a result of lower-value coefficients for backer satisfaction.

Most importantly though, we identified types of campaign information that have opposing relationships with fundraising and eventual backer satisfaction. Two concern contract design: the number of rewards and the funding target amount. These effects make sense when we treat fundraising as a competitive process between campaigns. If these campaigns amount to bidding for backer contracts, then the creators must reveal their expected costs and willingness to share risk as agents. More money for fewer rewards thus suggests creators are allocating more time and money for each contingent outcome. This is likely to increase the outcome quality but decrease perceptions of efficiency with their "bids."

The other types of information with opposing relationships with fundraising and eventual backer satisfaction concern deferred compensation. The number of past projects by creators was positively related to fundraising but negatively to eventual backer satisfaction. Meanwhile, the use of authentic language and a Facebook profile were negatively related to fundraising but positively to eventual backer satisfaction. Consider the roles of social identity and deferred compensation. From a backer's perspective, a creator signaling platform-specific identity suggests high loyalty and low likelihood of defecting, magnifying the long-term benefits and gains. Conversely, a creator signaling non-specific platform identity may suggest low loyalty and a high likelihood of defecting. Yet crowdfunding projects are fleeting by nature on platforms like Kickstarter. This means longterm social connections between creators and backers are generally reliant on connections and identities outside these platforms. Thus, signaling these non-specific platform identities is actually more likely to magnify the importance of deferred compensation obtained via reputational cost-benefit and emerging opportunities.

Finally, this study documents platform-level evidence that collective fundraising and collective backer satisfaction have shifted to exhibit opposing trajectories. Further, we found signs that this shift has coincided with deteriorating participation by backers on Kickstarter. Further analysis is required to unpack the nuances of these platform-level patterns, as well as how they relate to project-level incentive misalignment.

\section{Conclusion}

The main insight from this study is that rewardsbased crowdfunding may be unsustainable in its current form in the market mechanism that we investigated. We think this is true even though Kickstarter is one of the most-recognizable crowdfunding platforms. Indeed, it has established many of the fundraising dynamics for this type of crowdfunding and launched many high-profile technology and art projects. Yet systemic issues in its incentive structure seem to be contributing to a decline in campaign backers' collective satisfaction, as well as the number of projects getting funded on the platform. Surprisingly, the observed decline in satisfaction and participation coincide with Kickstarter's decision to lower restrictions on who can launch projects. This casts doubt on the likely impact of crowdfunding as an alternative channel in the future.

We contribute to research by: (1) by modeling creator-backer incentive alignment, as well as backer satisfaction - which represent a new empirical direction for the crowdfunding literature; and (2) demonstrating that it is possible to adopt an "inverse image" for empirical analysis, and move away from the over-emphasis on funds raised in crowdfunding projects. This will pave the way, we assert, for new theoretical perspectives to develop further enrich our knowledge in this area. The addition of backer satisfaction is also important for studies of the macro-impacts of crowdfunding. Causal connections between regional trends and the number of successful campaigns may be incomplete or even misleading without also modeling backers' reactions to outcomes in geospatial terms - a methods advance opportunity.

We contribute to practice with three potential remedies to address possible deterioration of rewards-based crowdfunding platforms. First, the platforms need to address the incentive misalignment at its core by adopting staggered payment models. This is becoming more common outside of Kickstarter, as platform like Patreon introduce subscription-based models and equity platforms 
use multiple (not single) funding rounds. Second, platforms may embed fundraising in other websites and social media used by creators. Creators will then be more likely to experience the long-term reputational costs and benefits related to with project outcomes. ${ }^{18}$

Third, platforms may provide more explicit structure as to what information creators should provide and how backers can offer more effective input. This will encourage information disclosure by creators and feedback from backers. Kickstarter has already added some of these features that projects must include (in its "Risks and Challenges" section) [24]. More could be done, ranging from additional creator disclosure sections to scheduled and automated backer polling.

Finally, we acknowledge several limitations in this research. First, we focused on a single platform with a disproportionately North American presence. So we could not learn the extent to which backer satisfaction may be affected by the extent of national regulation in the U.S., or perhaps the cultural effects that may be present. Second, our models produced notably lower coefficients for backer satisfaction than for fundraising success. An explanation is that our model drew on literature that aimed to find indicators that enhanced the likelihood of stronger fundraising, rather than backer satisfaction. Another reason, that backer satisfaction may be dependent on more factors, expresses their impacts as project development occurs over a longer period than fundraising.

Third, perhaps sentiment analysis does not yield a sensitive enough measure, so multiple methods can be used to create and compare other backer satisfaction measures. Fourth, this could be combined with other estimation techniques to examine temporal effects over the course of individual projects. Fifth, we are also working on quasi-experimental, causal-inference methods that enable innovative estimation and in-sample propensity-score matching methods to better align the datagenerating distributions for funding success and relate to the backers' characteristics $[19,35]$. At the conference, we expect to share progress toward a research design that permits causal inferences that more strongly support our findings.

Acknowledgments. We thank the HICSS Track and Minitrack Chairs and anonymous reviewers for insightful comments. R. Kauffman appreciated the support of the Endowed Chair in Digitalization at the Copenhagen Business School.

\section{References}

1. T. Beaulieu, S. Sarker, "Discursive meaning creation in crowdfunding: A socio-material perspective," in Proc. Intl. Conf. Sys., Sci., AIS Press, Atlanta, 2013.

\footnotetext{
${ }^{18}$ Projects like Star Citizen have used this approach to embed rewardsbased fundraising in their website, thus extending the funding period
}

2. P. Belleflamme, T. Lambert, A. Schwienbacher, "Crowdfunding: Tapping the right crowd,” J. Bus. Vent., 29(5), 2014, 585-609.

3. M. Benaroch, Y., Lichtenstein, L. Fink, "Contract design choices and the balance of ex ante and ex post transaction costs in software development outsourcing," MIS Qtrly., 40(1), 2016, 57-82.

4. D. Blaseg, C. Schulze, B. Skiera, "Consumer protection on Kickstarter," Mktg Sci., 2020, in press.

5. R.D. Boettger, C.R. Greer, "On the wisdom of rewarding A while hoping for B,” Org. Sci., 5(4), 1994, 569-582.

6. P. Booth, "Crowdfunding: A spimatic application of digital fandom,” New Media Soc., 17(2), 2015, 149-166.

7. C. Clogg, E. Petrova, A. Haritou, "Statistical methods for comparing regression coefficients between models," Amer. J. Soc., 100(5), 1995), 1261-1293.

8. B.L. Connelly, S.T. Certo, R.D. Ireland, C.R. Reutzel, "Signaling theory: A review and assessment," J. Mgmt., 37(1), 2011, 39-67.

9. C. Courtney, S. Dutta, Y. Li, "Resolving information asymmetry: Signaling, endorsement, and crowdfunding success,” Entrepr. Theory Prac., 41(2), 2017, 265-290.

10. D.J. Cumming, L. Hornuf, M. Karami, D. Schweizer, "Disentangling crowdfunding from fraudfunding," Max Planck Inst. for Innov. \& Compet. Res., Munich, 2020.

11. K.M. Eisenhardt, "Agency theory: An assessment and review,” Acad. Mgmt. Rev., 14(1), 1989, 57-74.

12. D. Frydrych, A.J. Bock, T. Kinder, B. Koeck, "Exploring entrepreneurial legitimacy in reward-based crowdfunding,” Vent. Cap., 16(3), 2014, 247-269.

13. R. Gibbons, K.L. Murphy, "Optimal incentive contracts in the presence of career concerns: Theory and evidence,” $J$. Pol. Econ., 100(3), 1992, 468-505.

14. R. Gleasure, K. Conboy, L. Morgan, “Talking up a storm: how backers use public discourse to exert control in crowdfunded systems development projects," Info. Sys. Res., 30(2), 2019, 447-465.

15. A.K. Gupta, V. Govindarajan, A. Malhotra, "Feedbackseeking behavior within multinational corporations," Strat. Mgmt. J., 20(3), 1999, 205-222.

16. H. Gurnani, M. Shi, "A bargaining model for a first-time interaction under asymmetric beliefs of supply reliability," Mgmt. Sci., 52(6), 2006, 865-880.

17. P.M. Healy, K.G. Palepu, "Information asymmetry, corporate disclosure, and the capital markets: A review of the empirical disclosure literature,” J. Acct. Econ., 31(1-3), 2001, 405-440.

18. Y. Hong, Y. Hu, G. Burtch, "Embeddedness, pro-sociality, and social influence: Evidence from online crowdfunding,” MIS Qtrly., 42(4), 2018, 1211-1224.

19. K.B. Karlson, A. Holm, and R. Breen, "Comparing regression coefficients between same-sample nested models using logit and probit: A new method." Soc. Meth., 42, 2012, 286-313.

20. K. Kim, I.H. Hann, "Crowdfunding and the democratization of access to capital-an illusion? Evidence from housing prices,” Info. Sys. Res., 30(1), 2019, 276-290.

21. M. Klein, A.C.B. Garcia, "High-speed idea filtering with

and building an integrated community. Wikipedia also uses websiteembedded donation-style fundraising for its periodic funding calls. 
the bag of lemons,” Dec. Supp. Sys., 78, 2015, 39-50.

22. D. Leone, F. Schiavone, L. Dezi, "Post-campaign operational problems hindering promised rewards in crowdfunding,” J. Innov. Econ. Mgmt., 2, 2018, 173-194.

23. G. Lewis, P. Bajari, "Moral hazard, incentive contracts, and risk: evidence from procurement," Rev. Econ. Stud., 81(3), 2014, 1201-1228.

24. T.P. Liang, S.P.J. Wu, C.C. Huang, "Why funders invest in crowdfunding projects: Role of trust from the dual-process perspective,” Info. \& Mgmt., 56(1), 2019, 70-84.

25. J.M. Madsen, J.L. McMullin, "Economic consequences of risk disclosures: Evidence from crowdfunding," Acct. Rev., 2020, in press.

26. G.P. Martin, R.M. Wiseman, L.R. Gomez-Mejia, “The interactive effect of monitoring and incentive alignment on agency costs,” J. Mgmt., 45(2), 2019, 701-727.

27. E. Mollick, R. Nanda, "Wisdom or madness? Comparing crowds with expert evaluation in funding the arts," Mgmt. Sci., 62(6), 2016, 1533-1553.

28. T.W. Moss, D.O. Neubaum, M. Meyskens, M., “The effect of virtuous and entrepreneurial orientations on microfinance lending and repayment: A signaling theory perspective,” Entrepr. Theory Prac., 39(1), 2015, 27-52.

29. S. Ryu, Y.G. Kim, "Money is not everything: A typology of crowdfunding project creators," J. Strat. Info. Sys., 27(4), 2018, 350-368.

30. F. Schiavone, "Incompetence and managerial problems delaying reward delivery in crowdfunding," J. Innov. Econ. Mgmt., 2, 2017, 185-207.

31. M. Szmigiera, "Crowdfunding market size globally 2019 and 2026,” Statista, Hamburg, Germany, September 22, 2020.

32. M.A. Stanko, D.H. Henard, "Toward a better understanding of crowdfunding, openness and the consequences for innovation,” Res. Pol., 46(4), 2017, 784-798.

33. M. Wessel, F. Thies, A. Benlian, "The emergence and effects of fake social information: Evidence from crowdfunding,” Dec. Supp. Sys., 90, 2016, 75-85.

34. M. Wessel, F. Thies, A. Benlian, "Opening the floodgates: The implications of increasing platform openness in crowdfunding,” J. Info. Tech., 32(4), 2017, 344-360.
35. R. Winkelmann, "Copula bivariate probit models: With an application to medical expenditures," Health Econ., 21, 2012, 1444-1455.

36. P. Younkin, V. Kuppuswamy, "The colorblind crowd? Founder race and performance in crowdfunding," Mgmt. Sci., 64(7), 2018, 3269-3287.

37. X. Zhang, K.M. Bartol, K.G. Smith, M.D. Pfarrer, D.M. Khanin, D. M., "CEOs on the edge: Earnings manipulation and stock-based incentive misalignment," Acad. Mgmt. J., 51(2), 2008, 241-258.

\section{Appendix Table A. Descriptive statistics}

\begin{tabular}{|c|c|c|}
\hline VARIABLE & MEAN & STD. DEV. \\
\hline \multicolumn{3}{|c|}{ Dependent variables } \\
\hline FundSucc & 0.421 & 0.494 \\
\hline BackerSatisf & 0.965 & 0.183 \\
\hline \multicolumn{3}{|l|}{ Contract design } \\
\hline FundGoal $^{\dagger}$ & 8.549 & 1.691 \\
\hline Duration & 33.093 & 11.574 \\
\hline TimeDeliv & 86.072 & 104.045 \\
\hline \#Rewards $^{\dagger}$ & 2.001 & 0.626 \\
\hline \multicolumn{3}{|c|}{ Information disclosure } \\
\hline Video & 0.704 & 0.456 \\
\hline \#Images $^{\dagger}$ & 1.244 & 1.263 \\
\hline Readability & 63.457 & 13.203 \\
\hline SpellingErrors & 0.090 & 0.287 \\
\hline \#Words ${ }^{\dagger}$ & 5.954 & 0.960 \\
\hline NumTerms & 2.836 & 2.410 \\
\hline \multicolumn{3}{|l|}{ Feedback } \\
\hline BackerComm $^{\dagger}$ & 0.788 & 1.344 \\
\hline BackerSenti & 0.208 & 0.317 \\
\hline \multicolumn{3}{|c|}{ Deferred compensation } \\
\hline \#Updates $^{\dagger}$ & 1.096 & 1.122 \\
\hline BackedOthers & 0.489 & 0.500 \\
\hline CreatorComm & 0.162 & 0.369 \\
\hline PastProj & 0.201 & 0.401 \\
\hline Authenticity & 24.321 & 20.719 \\
\hline FBProfile & 0.500 & 0.500 \\
\hline \multicolumn{3}{|c|}{ Control variables } \\
\hline US (0/1) & 0.718 & 0.450 \\
\hline StaffPick & 0.100 & 0.300 \\
\hline
\end{tabular}

